\title{
Sensitivity and specificity of serological tests, histopathology and immunohistochemistry for detection of Toxoplasma gondii infection in domestic chickens
}

\author{
L. Casartelli-Alves ${ }^{\mathrm{a}}$, V.C. Boechat ${ }^{\mathrm{a}}$, R. Macedo-Couto ${ }^{\mathrm{a}}$, L.C. Ferreira ${ }^{\mathrm{a}}$, \\ J.L. Nicolau ${ }^{\mathrm{b}}$, L.B. Neves ${ }^{\mathrm{b}}$, P.R. Millar ${ }^{\mathrm{c}}$, R.T. Vicente ${ }^{\mathrm{b}}$, R.V.C. Oliveira ${ }^{\mathrm{a}}$, \\ A.G. Muniz ${ }^{\text {d }}$, I.C.F. Bonna ${ }^{a}$, M.R.R. Amendoeira ${ }^{b}$, R.C. Silva ${ }^{\mathrm{e}}$, H. Langoni $^{\mathrm{f}}$, \\ T.M.P. Schubach ${ }^{a}$, R.C. Menezes ${ }^{a, *}$ \\ a Instituto Nacional de Infectologia Evandro Chagas, Fundação Oswaldo Cruz, Av. Brasil, 4365, Rio de Janeiro, RJ CEP: 21040-360, Brazil \\ b Instituto Oswaldo Cruz, Fundação Oswaldo Cruz, Av. Brasil, 4365, Rio de Janeiro, RJ CEP: 21040-360, Brazil \\ ' Instituto Biomédico, Universidade Federal Fluminense, Rua Professor Ernani Melo, 101, Niterói, RJ CEP: 24210-130, Brazil \\ d Instituto Nacional do Câncer, Praça da Cruz Vermelha, 23, Rio de Janeiro, RJ CEP: 20230-130, Brazil \\ e College of Veterinary Medicine, Mississippi State University, 240 Wise Center Drive, 39762 Starkville, USA \\ ${ }^{\mathrm{f}}$ Faculdade de Medicina Veterinária e Zootecnia, Universidade Estadual Paulista Júlio de Mesquita Filho, Botucatu, SP CEP: 18618-970, \\ Brazil
}

\section{A R T I C L E I N F O}

\section{Article history:}

Received 9 October 2013

Received in revised form 24 May 2014

Accepted 27 May 2014

\section{Keywords:}

Diagnosis

Toxoplasma gondii

Chickens

Serology

Histopathology

Immunohistochemistry

\begin{abstract}
A B S T R A C T
Since free-range chickens are important for the epidemiology of toxoplasmosis, this study evaluated the sensitivity and specificity of different laboratory techniques for the diagnosis of Toxoplasma gondii in these animals. Serum samples from 135 adult domestic chickens were tested for anti-T. gondii antibodies by the indirect fluorescent antibody test (IFAT), enzyme-linked immunosorbent assay (ELISA), modified agglutination test (MAT), and indirect hemagglutination test (IHAT). Tissue samples from all animals were analyzed by histopathology, immunohistochemistry and mouse bioassay (gold standard). Fifty-four chickens were positive for $T$. gondii in the bioassay. The sensitivity and specificity of the different tests were, respectively, $85 \%$ and $56 \%$ for ELISA; $80 \%$ and $52 \%$ for IFAT; $76 \%$ and $68 \%$ for MAT; $61 \%$ and $80 \%$ for IHAT; $7 \%$ and $98 \%$ for immunohistochemistry, and $6 \%$ and $98 \%$ for histopathology. The MAT was the most effective method for the diagnosis of T. gondii infection in chickens, followed by ELISA. Histopathology and immunohistochemistry are useful tools for the diagnosis of T. gondii infection in chickens due to their specificity.
\end{abstract}

(C) 2014 Elsevier B.V. All rights reserved.

\section{Introduction}

Toxoplasmosis is a worldwide zoonosis caused by the protozoan Toxoplasma gondii which affects mammals and

\footnotetext{
* Corresponding author. Tel.: +55 213865 9536; fax: +55 2138659553

E-mail addresses: rodrigo.menezes@ipec.fiocruz.br, rodrigo.menezesfiocruz@gmail.com (R.C. Menezes).
}

birds. Free-range chickens are good indicators of environmental contamination since they feed from the ground, exposing them to infection with $T$. gondii oocysts (Dubey et al., 2012). Another fact of epidemiological importance is that raw or undercooked chicken meat is an important source of infection for humans and other animals when it is consumed or handled without good hygiene (CasartelliAlves et al., 2012; Dubey et al., 2012; Hill and Dubey, 2013). 
Serological and molecular techniques, histopathology, immunohistochemistry and bioassays in mice or cats (gold standard) are used for the definitive diagnosis of $T$. gondii infection in chickens (Dubey et al., 2007; Yan et al., 2010; Dubey, 2010). Serological tests are frequently employed in epidemiological surveys and, like the polymerase chain reaction (PCR), are sensitive methods for the diagnosis of $T$. gondii infection in chickens (Dubey, 2010; Yan et al., 2010). The modified agglutination test (MAT) is the most commonly used method, whereas techniques such as the enzyme-linked immunosorbent assay (ELISA), indirect fluorescent antibody test (IFAT) and indirect hemagglutination test (IHAT) are used less frequently (Dubey, 2010). Although less sensitive, immunohistochemistry and histopathology permit visualization of the parasite and its correlation with histological alterations, which is not possible with serological tests or PCR.

The sensitivity and specificity of diagnostic methods for $T$. gondii infection using the mouse bioassay as the gold standard have been investigated in pigs (Dubey et al., 1995), but not in domestic chickens. In view of the epidemiological role of free-range chickens, an accurate diagnosis of $T$. gondii infection in these animals is very important. The more accurate this diagnosis, the more precise will be the evaluation of environmental contamination with $T$. gondii in the area studied. Therefore, the objective of the present study was to evaluate the sensitivity, specificity and accuracy of IFAT, ELISA, MAT, IHAT, immunohistochemistry and histopathology for the diagnosis of $T$. gondii infection in free-range chickens (Gallus gallus domesticus).

\section{Materials and methods}

\subsection{Samples}

A non-probabilistic sample of 54 chickens that tested positive for $T$. gondii in the mouse bioassay used as the gold standard was selected for this study. Sample size calculation was not possible due to the wide variety of diagnostic techniques used, the absence of suitable sensitivity values in the literature, costs, and technical feasibility. However, the sample size was sufficient to guarantee an expected mean sensitivity of the serological tests of $70 \%$, an absolute error of $10 \%$ at this proportion (range $60 \%-80 \%$ ), and a level of significance of $11 \%$. At the end of the study, the power of the sample (54 positive chickens) to calculate the difference in sensitivity between diagnostic techniques was evaluated considering the same level of significance as the sample size (11\%). To obtain these 54 chickens, 135 chickens were necropsied and their tissues were tested by mouse bioassay between April 2009 and July 2011. All of these 135 chickens were clinically healthy.

The 135 adult free-range chickens used in this study were raised on 48 farms in the state of Rio de Janeiro, Brazil. Blood was collected from these animals by puncture of the axillary vein for IFAT, ELISA, MAT and IHAT. Next, the animals were euthanized by cervical dislocation and necropsied. After coagulation, the blood samples were centrifuged at $1125 \times g$ and the serum was separated and stored at $-20^{\circ} \mathrm{C}$ until the time of analysis. During necropsy, samples were collected from the encephalon (cerebrum, cerebellum and brainstem), heart and thigh muscle for histopathology, immunohistochemistry and mouse bioassay. The tissue samples used for histopathology and immunohistochemistry were fixed in $10 \%$ buffered formalin and processed for embedding in paraffin (Carson and Hladick, 2009). Fresh tissue samples were used for the mouse bioassay.

\subsection{Indirect fluorescent antibody test}

The IFAT for detection of anti-T. gondii IgG antibodies was performed as described previously (Camargo, 1996) using $T$. gondii $\mathrm{RH}$ strain tachyzoites as antigen. Rabbit anti-chicken $\operatorname{IgY}(\operatorname{IgG})$ conjugated to fluorescein isothiocyanate (FITC) (Sigma-Aldrich, Brazil) was used. The sera were diluted to $1: 16,1: 64,1: 256,1: 1024$, and $1: 4096$ in $0.01 \mathrm{M} \mathrm{PBS}(\mathrm{pH} 7.2)$. The slides were examined under an YFL epifluorescence microscope (Nikon ${ }^{\circledR}$, USA). Positive and negative controls and a conjugate control were included in each battery of slides. Titers $\geq 16$ were defined as positive.

\subsection{Enzyme-linked immunosorbent assay}

ELISA for detection of anti-T. gondii IgG antibodies was performed with the BIOLISA Toxoplasmosis IgG ${ }^{\circledR}$ kit (Bioclin, Quibasa Química Básica, Brazil) according to manufacturer instructions. However, since this kit has been developed for detection of anti-T. gondii IgG antibodies in human serum or plasma, some adaptations were made for the diagnosis in chicken serum. For adaptation, the conjugate of the kit was replaced with anti-chicken IgG (whole molecule) peroxidase conjugate ${ }^{\circledR}$ (Sigma-Aldrich, Brazil) using a dilution of 1:30,000. Serum of chickens experimentally infected with $T$. gondii and serum of chickens positive by in-house ELISA (Millar et al., 2012) were used as positive controls. Serum of chickens that tested negative by in-house ELISA, IFAT and IHAT served as negative control. Readings equal to or higher than the cut-off value of the plate were considered to be positive.

\subsection{Modified agglutination test}

The MAT for detection of anti-T. gondii IgG antibodies was performed according to a previously described protocol (Desmonts and Remington, 1980). A suspension of $T$. gondii RH strain tachyzoites fixed in 6\% formalin was used as antigen (Silva et al., 2002). The antigen was diluted to $1: 16,1: 64,1: 256,1: 1024$ and $1: 4096$. Positive and negative controls were included in each microplate. Titers $\geq 16$ were defined as positive.

\subsection{Indirect hemagglutination test}

The IHAT for detection of anti-T. gondii IgG antibodies in chicken serum and in serum of mice used in the bioassay was performed with the Imuno-HAI Toxoplasmose ${ }^{\circledR}$ kit (Wama Diagnóstica, Brazil) according to manufacturer instructions. Positive and negative controls were included in each plate and the cut-off titer was 16. 


\subsection{Histopathology and immunohistochemistry}

For histopathology, two serial $5-\mu \mathrm{m}$ sections per encephalon, heart and thigh muscle tissue were cut from paraffin blocks, stained with hematoxylin-eosin (Carson and Hladick, 2009), and examined. For immunohistochemistry, one 5- $\mu \mathrm{m}$ section per encephalon, heart and thigh muscle tissue was cut from paraffin blocks, mounted on silanized slides, deparaffinized in xylene, and rehydrated in decreasing ethanol concentrations. Endogenous peroxidase was blocked by incubating the slides in a solution of $30 \%$ hydrogen peroxide and methanol ( $45 \mathrm{ml}$ hydrogen peroxide and $55 \mathrm{ml}$ methanol) for $40 \mathrm{~min}$ at room temperature. For antigen retrieval, the slides were incubated in citrate buffer, $\mathrm{pH} 6.0$, at $98^{\circ} \mathrm{C}$ for $30 \mathrm{~min}$ in a water bath. The Novo Link Max Polymer Detection System ${ }^{\circledR}$ (Novocastra, UK) was used for the detection of $T$. gondii according to manufacturer instructions. The slides were incubated with the primary polyclonal anti-T. gondii antibody (rabbit) (Cell Marque, USA) diluted to $1: 50$, overnight at $4{ }^{\circ} \mathrm{C}$. Histological sections containing tissue cysts and $T$. gondii tachyzoites were incubated with $1.5 \%$ bovine serum albumin as negative control and with polyclonal anti-T. gondii antibody (rabbit) as positive control.

\subsection{Mouse bioassay}

The mouse bioassay was performed according to a previously described protocol (Dubey, 1998). A pool of $20 \mathrm{~g}$ of brain, heart and thigh muscle collected from each chicken was triturated, homogenized, and digested with an acid pepsin solution ( $\mathrm{pH}$ 1.1-1.2). Five adult specific pathogen-free female Swiss Webster mice were inoculated intraperitoneally with $1 \mathrm{ml}$ of this homogenate obtained from each chicken (Dubey et al., 2006). A total of 675 mice weighing 20-35 g were inoculated. The control group consisting of one mouse per chicken (total of 135 mice) was inoculated intraperitoneally with $1 \mathrm{ml} 0.9 \%$ sterile saline.

After inoculation, the mice were monitored daily over a period of 45-50 days. Animals showing clinical signs of toxoplasmosis and those that were still alive at the end of the observation period were euthanized by intraperitoneal injection of an overdose of thiopental sodium and blood was collected by intracardiac puncture for the IHAT. Next, these mice were submitted to necropsy for microscopic tissue analysis (Dubey et al., 2006). The bioassay was defined as positive when microscopic analysis revealed the presence of protozoa that were consistent with $T$. gondii in tissues (tachyzoites or cysts) or in peritoneal exudate (tachyzoites), or when anti-T. gondii antibody titers were detected in serum by IHAT in at least one of five mice tested per chicken.

\subsection{Statistical analysis}

The data were entered using the EpiData 3.1 software and analyzed using the Statistical Package for the Social Sciences, version 16. The sensitivity, specificity and accuracy of the diagnostic techniques were calculated using the results of the mouse bioassay as the gold standard. The sensitivity, specificity and accuracy of the IFAT and MAT according to the different antibody titers used were compared to the gold standard to determine the best cut-off titer for the diagnosis of $T$. gondii infection in chickens. An $89 \%$ confidence interval was considered in all analyses.

\subsection{Ethical approval}

The study was approved by the Ethics Committee for Animal Use of FIOCRUZ (permit no. L-012/09).

\section{Results}

Fifty-four (40\%) of the 135 chickens studied were positive for $T$. gondii in the mouse bioassay. Using the mouse bioassay, the parasite was detected in mouse tissues for 39 (72\%) of these 54 positive chickens. In 15 (28\%) of the 54 positive chickens submitted to the mouse bioassay, the parasite could not be demonstrated in mouse tissues, but mice were seropositive for $T$. gondii by the IHAT. In the mouse bioassay of the 54 positive chickens, the number of positive mice among the five animals tested was one in the samples of 16 (30\%) chickens, two in the samples of four (7\%) chickens, three in the samples of eight (15\%) chickens, four in the samples of $12(22 \%)$ chickens and five in the samples of 14 (26\%) chickens. Neither T. gondii nor anti-T. gondii antibodies were detected in control animals of the mouse bioassays. Five ( $9 \%$ ) chickens with a positive bioassay result were negative in all serological and histological tests.

The power of the sample for comparing the sensitivity of the diagnostic techniques, considering 54 chickens with a positive bioassay result, was $99 \%$ for histopathology and immunohistochemistry compared to the serological techniques; 87\% for ELISA compared to IHAT; 68\% for IFAT compared to IHAT; $45 \%$ for MAT compared to IHAT; $25 \%$ for MAT compared to ELISA; $12 \%$ for ELISA compared to IFAT; $11 \%$ for MAT compared to IFAT; and 5\% for histopathology compared to immunohistochemistry.

Table 1 shows the sensitivity, specificity and accuracy of the different techniques used for the diagnosis of $T$. gondii in chickens. The sensitivity, specificity and accuracy of IFAT and MAT according to the different antibody titers used for the diagnosis of $T$. gondii infection in chickens are shown in Table 2.

Considering the 54 chickens with a positive result in the mouse bioassay, the false-positive rate was $48 \%$ (39/81) for IFAT, 44\% (35/80) for ELISA, 32\% (26/81) for MAT, $20 \%$ (16/79) for IHAT, 2\% (2/81) for histopathology, and $2 \%(2 / 80)$ for immunohistochemistry. The false-negative rate was $94 \%(51 / 54)$ for histopathology, 93\% (50/54) for immunohistochemistry, 39\% (21/54) for IHAT, 24\% (13/54) for MAT, 20\% (11/54) for IFAT, and 15\% (8/54) for ELISA.

Eight chickens were positive for $T$. gondii when the results of immunohistochemistry and histopathology were combined. Only tissue cysts were observed. Five of these animals had tissue cysts only in the heart (Fig. 1A and B), one only in the cerebellum, one only in the cerebrum, and another in the cerebrum and brainstem. There were no histological alterations associated with the presence of cysts. Three of the eight chickens with a positive histological result were negative in the mouse bioassay. 
Table 1

Sensitivity, specificity and accuracy of ELISA, IFAT, MAT, IHAT, immunohistochemistry and histopathology for the diagnosis of T. gondii infection in 135 chickens using a mouse bioassay as the gold standard.

\begin{tabular}{lll}
\hline Technique & \% Sensitivity $(89 \% \mathrm{CI})$ & \% Specificity $(89 \% \mathrm{CI})$ \\
\hline ELISA & $85(80-90)$ & $56(49-63)$ \\
IFAT & $80(74-85)$ & $52(45-59)$ \\
MAT & $76(70-82)$ & $68(61-74)$ \\
IHAT & $61(54-68)$ & $80(74-85)$ \\
Immunohistochemistry & $7(4-11)$ & $98(95-100)$ \\
Histopathology & $6(2-9)$ & $98(95-100)$ \\
\hline
\end{tabular}

ELISA: enzyme-linked immunosorbent assay; IFAT: indirect fluorescence antibody test; MAT: modified agglutination test; IHAT: indirect hemagglutination test; $89 \% \mathrm{CI}: 89 \%$ confidence interval.

Table 2

Sensitivity, specificity and accuracy of the IFAT and MAT according to the different antibody titers used for the diagnosis of $T$. gondii infection in 135 chickens using a mouse bioassay as the gold standard.

\begin{tabular}{|c|c|c|c|c|c|c|}
\hline \multirow[t]{2}{*}{ Titer } & \multicolumn{3}{|l|}{ IFAT } & \multicolumn{3}{|l|}{ MAT } \\
\hline & \% Se $(89 \% \mathrm{CI})$ & $\% \mathrm{Sp}(89 \% \mathrm{CI})$ & \%A (89\% CI) & $\% \mathrm{Se}(89 \% \mathrm{CI})$ & $\%$ Sp $(89 \%$ CI $)$ & $\% \mathrm{~A}(89 \% \mathrm{CI})$ \\
\hline 16 & $80(74-85)$ & $52(45-59)$ & $63(56-70)$ & $76(70-82)$ & $68(61-74)$ & $71(65-77)$ \\
\hline 64 & $69(62-75)$ & $74(68-80)$ & $72(66-78)$ & $50(43-57)$ & $94(91-97)$ & $76(70-82)$ \\
\hline 256 & $37(30-44)$ & $91(87-95)$ & $70(63-76)$ & $20(15-26)$ & $96(94-99)$ & $66(59-72)$ \\
\hline 1024 & $9(5-13)$ & $99(97-100)$ & $63(56-70)$ & $2(0-4)$ & $99(97-100)$ & $60(53-67)$ \\
\hline
\end{tabular}

Se: sensitivity; Sp: specificity; A: accuracy; IFAT: indirect fluorescence antibody test; MAT: modified agglutination test; 89\% CI: 89\% confidence interval.
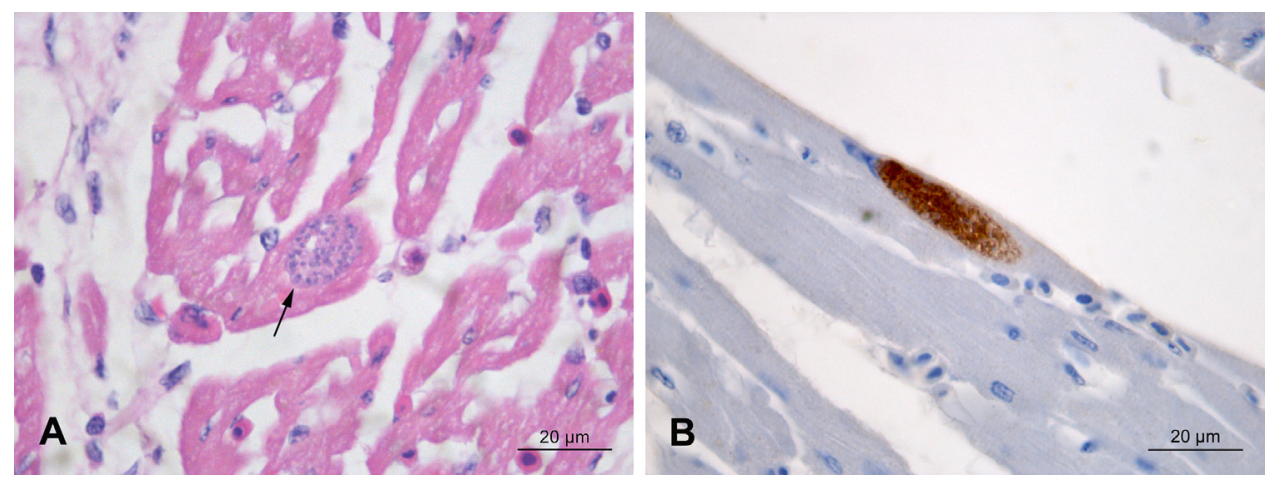

Fig. 1. (A) Histological section of the heart of a chicken showing a Toxoplasma gondii cyst (arrow); HE. (B) Immunohistochemistry of the heart of a chicken showing a Toxoplasma gondii cyst stained dark brown. (For interpretation of the references to color in this figure legend, the reader is referred to the web version of this article.)

Considering not only the mouse bioassay as the gold standard but also the histological tests, the sensitivity and specificity of the different serological tests evaluated were, respectively, $86 \%$ and $58 \%$ for ELISA, $81 \%$ and $54 \%$ for IFAT, $77 \%$ and $71 \%$ for MAT, and $63 \%$ and $83 \%$ for IHAT.

\section{Discussion}

Individual analysis of the diagnostic techniques showed that ELISA, followed by the IFAT, was the most sensitive method and IHAT was the most specific method for the serological diagnosis of $T$. gondii infection in chickens. Although the sensitivity of the MAT was lower than that of ELISA and IFAT, its accuracy was higher and very similar to that of IHAT. In addition, the MAT is a simple, low-cost method that does not use a specific conjugate, whereas IFAT and ELISA require the use of a species-specific conjugate and the interpretation of the IFAT result is subjective (Cañón-Franco et al., 2003; Millar et al., 2012). Therefore, the MAT is the most indicated method among those tested for the diagnosis of $T$. gondii infection in chickens in epidemiological surveys designed to detect areas of environmental contamination. These results confirm the efficacy of the MAT in the diagnosis of $T$. gondii infection in chickens demonstrated by other investigators (Silva et al., 2003; Dubey et al., 2006; Dubey, 2010; Beltrame et al., 2012). Although the most sensitive method, ELISA would be a second option since its specificity was one of the lowest among the diagnostic techniques studied. The IFAT was the second most sensitive diagnostic technique, but the least specific. Other investigators (Brandão et al., 2006) also reported limited specificity of the IFAT in detecting anti-T. gondii antibodies in chicken serum. The IHAT was found to be a sensitive and specific method in the present study, in agreement with other authors (Beltrame et al., 2012) who observed a sensitivity of $78 \%$ and specificity of $83 \%$ in relation to the MAT. In contrast, another study (Frenkel, 1981) reported low sensitivity and specificity of the IHAT ( $46 \%$ and $25 \%$, respectively) for the diagnosis of $T$. gondii in birds. The higher sensitivity and specificity of the IHAT observed here and in another study (Beltrame et al., 2012) are probably related to the fact that the same kit 
and cut-off value were used in those studies, whereas a different kit and cut-off value were adopted in the other study (Frenkel, 1981). Therefore, as suggested previously (Beltrame et al., 2012), the IHAT using the kit of the present study is an option for the diagnosis of $T$. gondii infection in chickens, particularly in situations in which the MAT, ELISA or IFAT cannot be carried out.

In the present study, all serological methods tested showed limited sensitivity and specificity in chickens. These values, except for the sensitivity of ELISA, were lower than those reported in another study (Dubey et al., 1995) for the serological diagnosis of $T$. gondii in sows. In that study, sensitivity and specificity were $82.9 \%$ and $90.2 \%$, respectively, for the MAT, the most sensitive method, and specificity was $98.3 \%$ for the IHAT, the most specific method. These differences might be related to the different animal species studied and to the fact that the other authors (Dubey et al., 1995) used bioassays in mice and cats as the gold standard, with the latter being more sensitive. However, comparison of the serological tests should be done with caution because of the limited power of the sample. Therefore, further studies involving a larger sample are needed for a more precise comparison of the different serological tests used for the diagnosis of $T$. gondii in chickens.

The occurrence of false-negative results of $T$. gondii infection in the serological tests evaluated here might be explained by the possibility of recent infection of these chickens when antibody titers are still not detectable. Chickens experimentally infected with $T$. gondii develop antibody titers detectable by MAT and IHAT on day 7 postinfection and by ELISA and IFAT on day 14 post-infection (Dubey et al., 1993; Sedlák et al., 2000; Yan et al., 2010). Another explanation would be a decline in the antibody titer of these animals to undetectable levels during chronic infection. The latter hypothesis, which has also been raised by other investigators for sows (Dubey et al., 1995), is more likely due to the fact that only adult birds were studied which could have been infected for many months or years. Other authors using the MAT (Silva et al., 2003) and IFAT (Brandão et al., 2006) also reported positive bioassay results in serologically negative chickens.

There were several cases of false-positive results of the serological tests. One possible explanation is crossreactivity with other parasites of the phylum Apicomplexa, such as Sarcocystis or Neospora which naturally infect freerange chickens (Munday et al., 1977; Martins et al., 2011). Cross-reactivity in serological tests has been observed between $T$. gondii and Neospora caninum in dogs (Silva et al., 2007) and between T. gondii and Sarcocystis in pigs (Moon, 1987; Lind et al., 1997). However, further studies on chickens are needed to confirm this hypothesis. Another explanation of the false-positive serological results in relation to the bioassay might be explained by failure of the latter technique. False-negative bioassay results can be due to the fact that the tissue samples of infected chickens used for this assay were not parasitized, contained non-viable parasites, or had a low parasite burden and were therefore unable to infect mice, a fact also reported for pigs (Dubey et al., 1995). Additionally, the tissue digestion step of the bioassay may reduce $T$. gondii viability (Dubey et al., 1995).
The three cases of chickens with a negative bioassay result in which $T$. gondii cysts were detected by histology confirm that the mouse bioassay is not $100 \%$ sensitive. As a consequence of this limited sensitivity of the mouse bioassay, the sensitivity and specificity of the serological tests evaluated were slightly underestimated. These underestimated values were demonstrated by the slight increase in the sensitivity and specificity of the serological tests evaluated when the histological tests were used as gold standard in addition to the mouse bioassay.

The sensitivity and specificity of the IFAT and MAT were also analyzed in the present study according to the different antibody titers used, since different cut-off values ranging from 8 to 40 for the IFAT and $\geq 5$ for the MAT (Dubey, 2010) have been reported for the diagnosis of $T$. gondii infection in chickens. A cut-off value $\geq 16$ used in the two tests showed the highest sensitivity in the present study and is recommended for the diagnosis of $T$. gondii infection in chickens.

In contrast to the serological techniques studied, histopathology and immunohistochemistry showed very low sensitivity and high specificity. It should be noted that the power of the sample for comparison of the histopathological and immunohistochemical results with the serological results was highly satisfactory. However, despite the similar specificity and sensitivity of histopathology and immunohistochemistry, the power of the sample was very low to confirm this similarity. The low sensitivity of histopathology and immunohistochemistry might be related to broad distribution of the protozoan in tissues (Tenter et al., 2000), a low parasite burden, and the small size of the tissue sample examined (5- $\mu \mathrm{m}$ sections). In contrast to the present study, $T$. gondii was not detected by histopathology in muscle or brain of infected pigs (Garcia et al., 2006). On the other hand, T. gondii was detected by immunohistochemistry in at least one organ (brain, liver, or heart) of 46 seropositive sheep (Silva et al., 2013). These results demonstrate that the concentration of tissue cysts of $T$. gondii per gram of brain or heart tissue in infected chickens may be higher than in infected pigs and lower than in infected sheep.

\section{Conclusions}

The MAT was the most effective method for the serological diagnosis of T. gondii infection in chickens. ELISA was also a sensitive method and could be a second option for use in serological surveys of chickens. Cut-off titers $\geq 16$ in both the MAT and IFAT were found to be effective for the diagnosis of $T$. gondii infection in chickens. Histopathology and immunohistochemistry are useful tools for the diagnosis of $T$. gondii infection in chickens due to their high specificity.

\section{Conflict of interest statement}

There is no conflict of interest.

\section{Acknowledgements}

This study was supported by Fundação Carlos Chagas Filho de Amparo à Pesquisa do Estado do Rio de Janeiro (FAPERJ) (grants APQ1 E-26/110.937/2009, APQ1 
E-26/111.977/2012, and TCT E-26/102.303/2011) and by Conselho Nacional de Desenvolvimento Científico e Tecnológico (CNPq) (grant MCT/CNPq 10/2010-AT-NM). TMPS has a research productivity fellowship from CNPq. RCM has a fellowship from FAPERJ (grant JCNE E-26/102.247/2013). We thank Eloíza P.F. Trindade, Maíra C.H. Cavalcanti, and Luiz C.S. Abboud for technical support; Dr. Fátima Madeira and the team of Laboratório de Vigilância em Leishmanioses, INI, FIOCRUZ, Brazil, for their collaboration with the study, and the histopathology technicians Francisco C.C. Rodrigues and Fernando F. Aguiar, INI, FIOCRUZ, for technical assistance.

\section{References}

Beltrame, M.A.V., Pena, H.F.J., Ton, N.C., Lino, A.J.B., Gennari, S.M., Dubey, J.P., Pereira, F.E.L., 2012. Seroprevalence and isolation of Toxoplasma gondii from free-range chickens from Espírito Santo state, southeastern Brazil. Vet. Parasitol. 188, 225-230.

Brandão, G.P., Ferreira, A.M., Melo, M.N., Vitor, R.W.A., 2006. Characterization of Toxoplasma gondii from domestic animals from Minas Gerais, Brazil. Parasite 13, 143-149.

Camargo, M.E., 1996. Toxoplasmose. In: Ferreira, A.W., Ávila, S.L.M. (Eds.), Diagnóstico laboratorial das principais doenças infecciosas e autoimunes. Guanabara Koogan, Rio de Janeiro, pp. 165-174.

Cañón-Franco, W.A., Bergamaschi, D.P., Richtzenhain, L.J., Nogueira, Y., Camargo, L.M.A., Souza, S.L.P., Gennari, S.M., 2003. Evaluation of the performance of the modified direct agglutination test (MAT) for detection of Toxoplasma gondii antibodies in dogs. Braz. J. Vet. Res. Anim. Sci. 40, 452-456

Carson, F.L., Hladick, C. (Eds.), 2009. Histotechnology: A Self-instructional Text. , 3rd ed. ASCP Press, Chicago, p. 400

Casartelli-Alves, L., Ferreira, L.C., Vicente, R.T., Millar, P.R., Oliveira, R.V.C., Amendoeira, M.R.R., Schubach, T.M.P., Menezes, R.C., 2012. Prevalência da infecção por Toxoplasma gondii em galinhas criadas extensivamente em Rio Bonito, Rio de Janeiro. Arq. Bras. Med. Vet. Zootec. 64, 1398-1401.

Desmonts, G., Remington, J.S., 1980. Direct agglutination test for diagnosis of Toxoplasma infection: method for increasing sensitivity and specificity. J. Clin. Microbiol. 11, 562-568.

Dubey, J.P., 1998. Refinement of pepsin digestion method for isolation of Toxoplasma gondii from infected tissues. Vet. Parasitol. 74, 75-77.

Dubey, J.P., 2010. Toxoplasma gondii infections in chickens (Gallus gallus domesticus): prevalence, clinical disease, diagnosis and public health significance. Zoonoses Public Health 57, 60-73.

Dubey, J.P., Ruff, M.D., Camargo, M.E., Shen, S.K., Wilkins, G.L., Kwok, O.C.H., Thulliez, P., 1993. Serologic and parasitologic responses of domestic chickens after oral inoculation with Toxoplasma gondii oocysts. Am. J. Vet. Res. 54, 1668-1672.

Dubey, J.P., Thulliez, P., Weigel, R.M., Andrews, C.D., Lind, P., Powell, E.C., 1995. Sensitivity and specificity of various serologic tests for detection of Toxoplasma gondii infection in naturally infected sows. Am. J. Vet. Res. 56, 1030-1036.

Dubey, J.P., Webb, D.M., Sundar, N., Velmurugan, G.V., Bandini, L.A., Kwok, O.C.H., Su, C., 2007. Endemic avian toxoplasmosis on a farm in Illinois: clinical disease, diagnosis, biologic and genetic characteristics of
Toxoplasma gondii isolates from chickens (Gallus gallus domesticus) and a goose (Anser anser). Vet. Parasitol. 148, 207-212.

Dubey, J.P., Gennari, S.M., Labruna, M.B., Camargo, L.M., Vianna, M.C., Marcet, P.L., Lehmann, T., 2006. Characterization of Toxoplasma gondii isolates in free-range chickens from Amazon, Brazil. J. Parasitol. 92, $36-40$.

Dubey, J.P., Lago, E.G., Gennari, S.M., Su, C., Jones, J.L., 2012. Toxoplasmosis in humans and animals in Brazil: high prevalence, high burden of disease, and epidemiology. Parasitology 139, 1375-1424.

Frenkel, J.K., 1981. False-negative serologic test for Toxoplasma in birds. J. Parasitol. 67, 952-953.

Garcia, J.L., Gennari, S.M., Machado, R.Z., Navarro, I.T., 2006. Toxoplasma gondii: detection by mouse bioassay, histopathology, and polymerase chain reaction in tissues from experimentally infected pigs. Exp. Parasitol. 113, 267-271.

Hill, D.E., Dubey, J.P., 2013. Toxoplasma gondii prevalence in farm animals in the United States. Int. J. Parasitol. 43, 107-113.

Lind, P., Haugegaard, J., Wingstrand, A., Henriksen, S.A., 1997. The time course of the specific antibody response by various ELISAs in pigs experimentally infected with Toxoplasma gondii. Vet. Parasitol. 71, $1-15$.

Martins, J., Kwok, O.C.H., Dubey, J.P., 2011. Seroprevalence of Neospora caninum in free-range chickens (Gallus domesticus) from the Americas. Vet. Parasitol. 182, 349-351.

Millar, P.R., Alves, F.M.X., Teixeira, V.Q., Vicente, R.T., Menezes, E.M., Sobreiro, L.G., Pereira, V.L.A., Amendoeira, M.R.R., 2012. Occurrence of infection with Toxoplasma gondii and factors associated with transmission in broiler chickens and laying hens in different raising systems. Pesq. Vet. Bras. 32, 231-236.

Moon, M.H., 1987. Serological cross-reactivity between Sarcocystis and Toxoplasma in pigs. Korean J. Parasitol. 25, 188-194.

Munday, B.L., Humphrey, J.D., Killa, V., 1977. Pathology produced by, prevalence of, and probable life-cycle of a species of Sarcocystis in the domestic fowl. Avian Dis. 21, 696-703.

Silva, A.F., Oliveira, F.C., Leite, J.S., Mello, M.F., Brandão, F.Z., Leite, R.I., Frazão-Teixeira, E., Lilenbaum, W., Fonseca, A.B., Ferreira, A.M., 2013. Immunohistochemical identification of Toxoplasma gondii in tissues from modified agglutination test positive sheep. Vet. Parasitol. 191, 347-352.

Silva, A.V., Cutolo, A.A., Langoni, H., 2002. Comparação da reação de imunofluorescência indireta e do método de aglutinação direta na detecção de anticorpos anti-Toxoplasma em soros de ovinos, caprinos, caninos e felinos. Arq. Inst. Biol. (Sao Paulo) 69, 7-11.

Silva, D.A.O., Lobato, J., Mineo, T.W.P., Mineo, J.R., 2007. Evaluation of serological tests for the diagnosis of Neospora caninum infection in dogs: optimization of cut off titers and inhibition studies of cross-reactivity with Toxoplasma gondii. Vet. Parasitol. 143, 234-244.

Sedlák, K., Literák, I., Vitula, F., Benák, J., 2000. High susceptibility of partridges (Perdix perdix) to toxoplasmosis compared with other gallinaceous birds. Avian Pathol. 29, 563-569.

Silva, D.S., Bahia-Oliveira, L.M.G., Shen, S.K., Kwok, O.C.H., Lehman, T., Dubey, J.P., 2003. Prevalence of Toxoplasma gondii in chickens from an area in southern Brazil highly endemic to humans. J. Parasitol. 89, 394-396.

Tenter, A.M., Heckeroth, A.R., Weiss, L.M., 2000. Toxoplasma gondii: from animals to humans. Int. J. Parasitol. 30,1217-1258.

Yan, C., Yue, C.L., Yuan, Z.G., Lin, R.Q., He, Y., Yin, C.C., Xu, M.J., Song, H.Q., Zhu, X.Q., 2010. Molecular and serological diagnosis of Toxoplasma gondii infection in experimentally infected chickens. Vet. Parasitol. $173,179-183$ 